\section{Profi-Schwimmtraining verändert funktionelle Schulteranatomie bei Jugendlichen}

Hibberd EE et al. Effect of Swim Training on the Physical Characteristics of Competitive Adolescent Swimmers. Am J Sports Med 2016; 44: 28132819

Schwimmen ist eine äußerst trainingsintensive Sportart, oft wird für bis zu 11 Monate im Jahr fast täglich trainiert, sodass der beanspruchten Muskulatur wenig Zeit zur Erholung bleibt. Häufige Folge sind chronische Schmerzen und Überlastung, die ein Training nur noch unter Schmerzmitteleinnahme erlauben. Mediziner aus den USA haben die Auswirkungen des Trainings auf anatomische Veränderungen in der Schulter untersucht.

Elizabeth Hibberd und ihre Kollegen haben 43 jugendliche Profischwimmer im Alter zwischen 13 und 18 Jahren in eine prospektive Beobachtungsstudie aufgenommen. Als Kontrollgruppe dienten 29 gleichaltrige Profisportler aus Fußball und Leichtathletik. Die Mediziner untersuchten die Sportler zu 3 Zeitpunkten:

- vor Beginn der Schwimmtrainingsaison,

- 6 Wochen nach Trainingsbeginn und

- 12 Wochen nach Trainingsbeginn.

Dabei untersuchten die Wissenschaftler jedes Mal

- die Position des Kopfes,

- die Position der Schulter, jeweils auf seitlichen Fotos relativ zum 7. Halswirbel, und

- die Breite des subakromialen Raums in der Sonografie, gemessen als kürzester Abstand zwischen der anterior-inferioren Spitze des Akromions und dem Humeruskopf.

Dann beurteilten sie die Veränderungen im Verlauf der Trainingswochen gegenüber den Ausgangswerten. Dabei fanden sich

- bei den Schwimmern nach Trainingsbeginn signifikant stärkere Veränderungen der Schulterhaltung, mit einer Vorwärtsverschiebung um ca. 15\%, während es bei den Kontrollsportlern nur knapp $1 \%$ war.

- Die Position des Kopfes verschob sich dagegen bei allen Sportlern nach vorne, unabhängig von der Sportart (7,2\% nach 12 Wochen bei den Schwimmern und $6 \%$ bei den Kontrollen).

- Der subakromiale Raum schließlich wurde bei den Schwimmern signifikant enger, mit einer Abnahme um 10,7\% am dominanten Arm; während sich in der Kontrollgruppe eine Zunahme um $1,5 \%$ fand.

Dabei zeigte sich eine moderate negative Korrelation der Veränderung der Schulterposition mit der Verengung des subakromialen Raums in Woche 6 (Pearson-Korrelationskoeffizient $r=-0,49$ ).

\section{FAZIT}

Die hier beobachteten anatomischen Veränderungen bei den Schwimmern könnten in der Folge zu einem verstärkten Kontakt zwischen Akromion und Rotatorenmanschettensehnen führen, so die Autoren. Letztlich kann daraus ein manifestes Schulterimpingement resultieren. Ursache könnte eine zu hohe Trainingsbelastung gerade in den Wochen nach der Ruhesaison sein. Diese Befunde betonen die Bedeutung eines wirksamen Präventionsprogramms, um Schmerzen und möglicherweise irreversible Schäden zu verhindern.

Dr. Elke Ruchalla, Bad Dürrheim 